\title{
Our panel of experts highlight the most important research articles across the spectrum of topics relevant to the field of pediatric brain tumors
}

Expert panel: Larry Junck, University of Michigan Health System, Ml, USA; Maura Massimino, Fondazione Istituto di Ricovero \& Cura a Carattere Scientifico Istituto Nazionale dei Tumori, Milan, Italy

First draft submitted: 3 October 2015; Accepted for publication: 13 October 2015; Published online: 18 December 2015

Wakefield A, Pignata A, Ghazi A et al. Is CMV a target in pediatric glioblastoma? Expression of CMV proteins, pp65 and IE1-72 and CMV nucleic acids in a cohort of pediatric glioblastoma patients. J. Neurooncol. 125(2), 307-315 (2015).

The interest on cytomegalovirus (CMV) correlation with adult glioblastoma has been high in the last few years. Human CMV proteins and nucleic acids are present in the majority of adult GBM and this occurrence has caused interest as possible target for immune-based therapies. This paper refers on the search of CMV through immunohistochemistry and in situ hybridization in 25 pediatric glioblastoma partly evaluated at diagnosis and partly after relapse. Both the antigens pp65 and IE1-72 were detected, according to the technique, in over $60 \%$ of the samples examined. These results highlight, at least from this point of view, a similarity between adult and pediatric glioblastoma. The hypothesis is that the ubiquitous CMV proteins identified in glioblastoma contribute to the malignant phenotype of glioblastoma. The authors interestingly conclude that further investigations on how these antigens can promote tumor growth and prevent cell death are warranted also in children.

- Written by Maura Massimino

Chamdine O, Broniscer A, Wu S, Gajjar

A, Qaddoumi I. Metastatic low-grade gliomas in children: 20 years' experience at St. Jude Children's Research Hospital. Pediatr. Blood Cancer doi:10.1002/ pbc.25731 (2015) (Epub ahead of print). Low grade glioma (LGG) can disseminate along the neuraxis, either at diagnosis or more frequently at the time of disease progression. This phenomenon was described in the literature long before the magnetic resonance imaging era. However, limited data are available on the true incidence, presentation, patterns of dissemination, disease behavior, outcome and best management approaches to treating metastatic LGG. This paper describes the biggest series of such entities both at diagnosis (19 patients) and at relapse (other 19 cases) diagnosed and treated between 1990 and 2010. The majority of patients had a diagnosis of pilocytic astrocytoma and treatment varied a lot during time both for the different extension of disease and for the different treatment lines that the patients received. At a median follow-up of 80 months, the long-term outcome was not very satisfying with a median survival of 6.2 years. It is to be remarked that while the 5 -year OS was positive, with over $80 \%$, it dropped at 10 and 15 years reaching $50 \%$ and thus showing a pattern of aggressiveness during the chronic time of the disease. In the vast and heterogenous world of LGG, the metastatic presentation represents even today a difficult and mysterious prognosis disease that needs more attention.

- Written by Maura Massimino

Dunham C. Uncommon pediatric tumors of the posterior fossa: pathologic and molecular features. Childs Nerv. Syst. 31, 1729-1737 (2015).

Dunham brings to our attention the existence of recently described pathologic entities arising in children posterior fossa other than pilocytic astrocytoma (PA), medulloblastoma, and ependymoma. The five less common tumors that may affect the posterior fossa of children are:
CNS Oncology

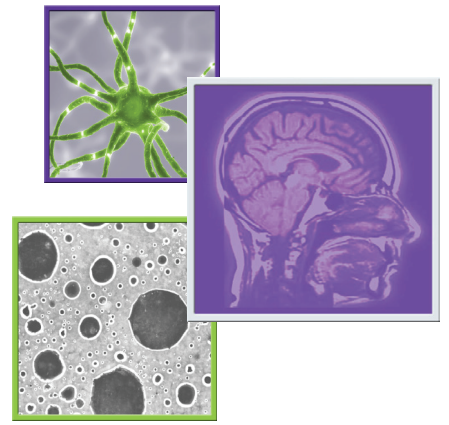

KEYWORDS

- ATRX

- co-deletion of 1p19q

- glioma • gliomagenesis

- isocitrate dehydrogenase

- low-grade glioma

- neuropathological

classification • TERT•TP53 
'embryonal tumor with multilayered rosettes' (ETMR); 'cribriform neuroepithelial tumor' (CRINET); 'rosette forming glioneuronal tumor' (RGNT); 'diffuse pilocytic astrocytoma' (dPA) and, 'desmoplastic small round cell tumor' (DSRCT). The most exciting observation, in this new genomic era, is that the molecular pathology of many, if not all, of the tumors highlighted herein, exhibit clearly defined genetic alterations. The most frequent entity of the five described, whose collective median overall survival is dismal and around 1 year, is the ETMR. Under the embryonal tumor with multilayered rosettes (ETMR) umbrella are three well-recognized histologic entities: ependymoblastoma (EBL); 'embryonal tumor with abundant neuropil and true rosettes' (ETANTR) and medulloepithelioma (MEPL). Each tumor is populated, to varying degrees, by primitive and highly mitotically active tumor cells and may additionally display distinctive true rosettes wherein a central lumen is surrounded by multiple layers of primitive tumor cells. Amplification of the miRNA cluster $\mathrm{C} 19 \mathrm{MC}$ at $19 \mathrm{q} 13.42$ is the key genetic alteration linking together the entities comprising ETMR. Gene expression profiling of greater than 1400 brain tumors later uncovered LIN28A to be a very sensitive and specific marker of ETMR.

The diffuse variant of the PA harboring BK fusion is another interesting entity because it is mostly represented in adult but, when described in children, shares with the adult compart a much less favorable outcome than classic pilocytic astrocytoma and deserves the treatment of a high-grade glioma.

The author correctly concludes that the identification of characteristic molecular alterations in these tumors help avert misdiagnoses and will be quickly become a mainstay in the clinicopathologic workup of these cases.

- Written by Maura Massimino

\section{Cancer Genome Atlas Research Network, Brat DJ, Verhaak RG et al. Comprehensive, integrative genomic analysis of diffuse lower-grade gliomas. New Engl. J. Med. 372(26), 2481-2498 (2015).}

The authors carried out genome-wide analyses, including exome sequencing and DNA copynumber profiling, on 293 grade II and III gliomas from adults. Unsupervised clustering revealed three robust subtypes. Gliomas with co-deletion of $1 \mathrm{P} / 19 \mathrm{Q}$ and $I D H$ mutation were more likely to occur in frontal lobe and were associated with median survival 8.0 years. Of these tumors, $96 \%$ carried activating TERT promoter mutations, and mutations were also common in $C I C$, PI3 kinase pathway genes, NOTCH1, and FUBP1. Gliomas with $I D H$ mutation but no co-deletion had median survival 6.3 years; $93 \%$ had TP53 mutation with presumed loss of function, and $86 \%$ had inactivating alterations in $A T R X$. Gliomas with wild-type $I D H$ most commonly arose in temporal lobe, and median survival was only 1.7 years. These tumors had a median of 45 mutations, compared with 27 and 28 in the other two classes, and $68 \%$ had TERT promoter mutation. They harbored genetic changes typical of glioblastoma, including PTEN mutation and chromosome 7 gain and 10 deletion. The wealth of data in this article strongly corroborate this simple new molecular classification over histopathological classification.

\section{- Written by Larry Junck}

\section{Eckel-Passow JE, Lachance DH, Molinaro AM et al. Glioma groups based on 1p/19q, IDH and TERT promoter mutations in tumors. New Engl. J. Med. 372, 2499-2508 (2015).}

Among 615 gliomas of grade II or III, 29\% were found to be 'triple-positive' with mutations of TERT promoter and IDH and codeletions in $1 \mathrm{p} / 19 \mathrm{q}, 5 \%$ had mutations in TERT promoter and $I D H$ only, $45 \%$ had mutations in $I D H$ only and $10 \%$ in TERT only, $7 \%$ were triple-negative and $5 \%$ had other combinations of these markers. These groupings were strongly prognostic for survival; compared with triple-positive tumors, multivariate hazard ratios for death was not significant for TERT plus $I D H$ (1.31), but was significantly unfavorable for $I D H$ only (2.08), triple negative (3.74) and TERT only (11.74). Among grade IV tumors, $74 \%$ had mutations in TERT only, $7 \%$ were mutated in $I D H$ only, $17 \%$ were triple-negative and the remaining few had other combinations of these markers. For grade IV gliomas, survival among the groups did not differ significantly by multivariate analysis. From these and other findings, the authors conclude that patient groups whose gliomas have various sets of these markers differ in ages of onset, survival, associations with germline variants and mechanisms of pathogenesis.

\section{- Written by Larry Junck}


Grasso CS, Tang Y, Truffaux $\mathrm{N}$ et al. Functionally defined therapeutic targets in diffuse intrinsic pontine glioma. Nat. Med. 21, 827 (2015).

Diffuse intrinsic pontine glioma (DIPG) is known to have a high frequency of K27M mutations in genes encoding histone $\mathrm{H} 3$, resulting in global hypomethylation of H3 Lys27 (H3K27). Research has been hampered by unavailability of tumor tissue, cell lines and model systems. Studying 14 patient-derived DIPG cell cultures, the authors tested 83 drugs for cell viability at $72 \mathrm{~h}$ and found 14 to demonstrate activity. The multi-HDAC inhibitor panobinostat was among the most effective. With panobinostat treatment, cell lines expressing the H3.3K27M mutation demonstrated a dose-dependent increase in $\mathrm{H} 3$ acetylation and $\mathrm{H} 3 \mathrm{~K} 27$ trimethylation, suggesting that the drug partially rescues the hypotrimethylation phenotype. Testing of panobinostat treatment in a mouse pontine DIPG xenograft model by intratumoral convection enhanced delivery showed inhibition of tumor growth. Combination testing with the histone demethylase inhibitor GSK-J4 demonstrated synergistic effects. The results suggest a promising strategy for treatment of DIPG.

- Written by Larry Junck

Financial \& competing interests disclosure

The authors have no relevant affiliations or financial involvement with any organization or entity with a financial interest in or financial conflict with the subject matter or materials discussed in the manuscript. This includes employment, consultancies, honoraria, stock ownership or options, expert testimony, grants or patents received or pending, or royalties.

No writing assistance was utilized in the production of this manuscript. 